\title{
Safety Evaluation of Physical Human-Robot Interaction via Crash-Testing
}

\author{
Sami Haddadin, Alin Albu-Schäffer, Gerd Hirzinger \\ Institute of Robotics and Mechatronics \\ DLR - German Aerospace Center \\ P.O. Box 1116, D-82230 Wessling, Germany \\ \{sami.haddadin, alin.albu-schaeffer, gerd.hirzinger\}@dlr.de
}

\begin{abstract}
The light-weight robots developed at the German Aerospace Center (DLR) are characterized by their low inertial properties, torque sensing in each joint and a load to weight ratio similar to humans. These properties qualify them for applications requiring high mobility and direct interaction with human users or uncertain environments. An essential requirement for such a robot is that it must under no circumstances pose a threat to the human operator. To actually quantify the potential injury risk emanating from the manipulator, impact test were carried out using standard automobile crash-test facilities at the ADAC ${ }^{1}$. In our evaluation we focused on unexpected rigid frontal impacts, i.e. injuries e.g. caused by sharp edges are excluded. Several injury mechanisms and so called Severity Indices are evaluated and discussed with respect to their adaptability to physical human-robotic interaction.
\end{abstract}

\section{INTRODUCTION \& MOTIVATION}

The desired coexistence of robotic systems and humans in the same physical domain, by sharing the same workspace and actually cooperating in a physical manner, poses the very fundamental problem of ensuring safety to the user and the robot.

Safety in terms of industrial robots usually consists of isolating the workspace of the manipulator from the one of human beings by a safety guard with locked safety doors or light barriers [1]. Once the safety door is opened or the light barrier is crossed, the robot is stopped immediately.

On the other hand an increasing interest has recently been observed in domestic and industrial service robots, characterized by desirable, and under certain circumstances even unavoidable physical interaction [2], [3], [4]. Therefore, a resulting essential requirement is to guarantee safety for human users in regular operation mode as well as in possible fault modes.

This requirement necessitates a quantification of potential danger by an objective measure of injury severity. Once it is possible to correlate the behavior of the robot with an estimation of the resulting injury, it has to be guaranteed that the actions of the robot cannot cause an exceedance of a safe maximum value if physical contact with the robot occurs.

According to ISO-10218, which defines new collaborative operation requirements for industrial robots [5], one of the following conditions always has to be fulfilled: The $\mathrm{TCP}^{2} /$ flange

\footnotetext{
${ }^{1}$ German Automobile Club

${ }^{2}$ Tool Center Point
}

velocity needs to be $\leq 0.25 \mathrm{~m} / \mathrm{s}$, the maximum dynamic power $\leq 80 \mathrm{~W}$, or the maximum static force $\leq 150 \mathrm{~N}$. However, these values are not derived from real human impact experiments or any biomechanical analysis but base on heuristics, intending to give a human the possibility to actively avoid dangerous situations. Up to now there do not exist any injury indices tailored to the needs of robotics and those borrowed from other fields (e.g. the Head Injury Criterion (HIC) [6], [7]) were mainly evaluated in simulation so far. Further approaches are outlined in [8], [9], [10], but the importance of human biomechanics was barely investigated. To fill this gap we decided to measure the potential danger emanating from the DLR lightweight robot III (LWRIII) by impact tests at a certified crash-test facility. These tests were conducted at the Crash-Test Center of the German Automobile Club ADAC. The robot was commanded to move on a predefined trajectory and hit various dummy body parts at TCP velocities up to $2 \mathrm{~m} / \mathrm{s}$. Based on the outcome of these experiments we can draw some general conclusions related to potential danger of robots, depending on their mass and velocity.

In our evaluation we concentrated on unexpected impacts of a smooth surface related to the three body regions head, neck, and chest. Injury mechanisms caused by sharp tools or similar injury sources were not taken into consideration, since these mechanisms cannot be measured with standard crash-test dummies. To evaluate the resulting injury severity the European testing protocol EuroNCAP was applied. The results of several injury criteria for head, neck, and chest were measured by the ADAC and will be presented in the paper. Because an overview of commonly used Severity Indices is missing in robotics, a short presentation on them will be given as well. The most prominent index for the head is the Head Injury Criterion [11] which was already introduced to robotics in [6], [7] and used as a basis for new actuation concepts [12], [13].

As mentioned above, work that has been carried out up to now in the field of physical human-robot interaction was mainly based on simulations. These contributions indicated high potential injury of humans by means of HIC, already at a robot speed of $1 \mathrm{~m} / \mathrm{s}$. This also perfectly matched to the "common sense" expectation that a robot moving at maximal speed (e.g. due to malfunction) can cause high impact injury. In this sense the paper presents very surprising and striking 
results.

Moreover, one of the main contributions of this paper is the first experimental evaluation of HIC in standard crashtest facilities. Additionally to the impact evaluation it will be shown that even with an ideally fast (physical) collision detection one is not able to react fast enough to a stiff collision (e.g. head) in order to decrease the effect of the contact forces for link inertias similar or larger to the ones of the LWRIII.

In Section II the evaluated injury limits and measures are defined and briefly explained, followed by the description of the testing setup in Section III. Consecutively, experimental results are presented in Section IV. The following evaluation and discussion lead to a number of surprising and quite general conclusions outlined in Section V.

\section{Classifying Injury Severity}

Before actually introducing the definition of evaluated Severity Indices, an intuitive and internationally established definition of injury level will be given.

\section{A. The Abbreviated Injury Scale}

\begin{tabular}{cll}
\hline AIS & SEVERITY & TYPE OF INJURY \\
\hline 0 & None & None \\
1 & Minor & Superficial Injury \\
2 & Moderate & Recoverable \\
3 & Serious & Possibly recoverable \\
4 & Severe & Not fully recoverable without care \\
5 & Critical & Not fully recoverable with care \\
6 & Fatal & Unsurvivable \\
\hline
\end{tabular}

TABLE I

Definition of the AbBREviated InJuRy SCALE.

A definition of injury level developed by the $\mathrm{AAAM}^{3}$ and the $\mathrm{AMA}^{4}$ is the Abbreviated Injury Scale (AIS) [14]. It subdivides the observed level of injury into seven categories from none to fatal and provides a very intuitive classification (see Tab.I). Of course this classification gives no hint how to measure possible injury, this is provided by so called Severity Indices.

\section{B. EuroNCAP}

The ADAC crash-tests are carried out according to the EuroNCAP $^{5}$ which is based on the Abbreviated Injury Scale. The EuroNCAP, inspired by the American NCAP, is a manufacturer independent crash-test program uniting the European ministries of transport, automobile clubs and underwriting associations with respect to their testing procedures and evaluations [15]. The outcome of the tests, specified in the program, is a scoring of the measured results via a sliding scale system. Upper and lower limits for the injury potentials are mostly defined such that they correlate to a certain probability

\footnotetext{
${ }^{3}$ Association for the Advancement of Automotive Medicine

${ }^{4}$ American Medical Association

${ }^{5}$ European National Car Assessment Protocol
}

of AIS $\geq 3$ (see e.g. Fig.5,6). Between these two values the corresponding score (injury potential) is calculated by linear interpolation. A standardized color code indicates injury potential and is given in Tab.II.

\begin{tabular}{lll}
\hline Colorcode & Color & Injury potential \\
\hline Red & & Very high \\
Brown & High \\
Orange & Medium \\
Yellow & & Low \\
Green & & Very low \\
\hline
\end{tabular}

TABLE II

INJURY SEVERITY AND CORRESPONDING COLOR CODE.

Since standard dummy equipment enables the measurement of Severity Indices for the head, neck and chest, those ones evaluated by our tests at the crash-test facilities will now be defined.

\section{Injury Criteria For The Head}

According to [16] most research carried out in connexions with automobile crash-testing distinguishes two types of head loadings:

1) Direct Interaction: An impact or blow involving a collision of the head with another solid object at appreciable velocity. This situation is generally characterized by large linear accelerations and small angular accelerations during the impact phase.

2) Indirect Interaction: An impulse loading including a sudden head motion without direct contact. The load is generally transmitted through the head-neck junction upon sudden changes in the motion of the torso and is associated with large angular accelerations of the head.

Since the potential danger is disproportionately higher by direct interaction, this work will concentrate on the first potential injury source.

Especially for the head quite many criteria for type 1 interactions are available. Their major theoretical basis is the so called $\mathrm{WSTC}^{6}$, a fundamental experimental injury tolerance curve forming the underlying biomechanical data of all presented head criteria. The limit values of the following injury criteria are defined in the EuroNCAP protocol. For the head they represent a $5 \%$ probability of occurring AIS $\geq 3$ injury.

1) Head Injury Criterion: The most frequently used head Severity Index is the Head Injury Criterion [11], defined as

$$
\begin{array}{r}
\mathrm{HIC}_{36}=\max _{\Delta t}\left\{\Delta t\left(\frac{1}{\Delta t} \int_{t_{1}}^{t_{2}}\left\|\ddot{\mathbf{x}}_{H}\right\|_{2} \mathrm{~d} t\right)^{\left(\frac{5}{2}\right)}\right\} \leq 650 \\
\Delta t=t_{2}-t_{1} \leq \Delta t_{\max }=36 \mathrm{~ms} .
\end{array}
$$

$\left\|\ddot{\mathbf{x}}_{H}\right\|$ is the resulting acceleration of the human head ${ }^{7}$ and has to be measured in $\mathrm{g}=9.81 \mathrm{~m} / \mathrm{s}^{2}$. The optimization

\footnotetext{
${ }^{6}$ Wayne State Tolerance Curve

${ }^{7}|| \ddot{\mathbf{x}} \|_{2}=$ Euclidean norm
} 
is done by varying $t_{1}$ and $t_{2}$, i.e. the start and stop time are both parameters of the optimization process. Intuitively speaking, the HIC weights the resulting head acceleration and impact duration, which makes allowance of the fact that the head can be exposed to quite high accelerations and is still intact as long as the impact duration is kept low. In addition to the $\mathrm{HIC}_{36}$ the identically defined $\mathrm{HIC}_{15}$ with $\Delta t_{\max }=15 \mathrm{~ms}$ exists. Comparing both likelihood distributions yields that corresponding injury probabilities for $\mathrm{HIC}_{15}$ are more restrictive than for the $\mathrm{HIC}_{36}$ (see Sec.II-C.3).

2) 3ms-Criterion:

$$
a_{3 \mathrm{~ms}}=\frac{1}{\Delta t} \int_{t_{1}}^{t_{2+\Delta t}}\left\|\ddot{\mathrm{x}}_{H}\right\|_{2} \mathrm{~d} t \leq 72 \mathrm{~g}, \quad \Delta t=3 \mathrm{~ms}
$$

This criterion requires the maximum 3ms-average of the resulting acceleration to be less than $72 \mathrm{~g}$. Any shorter impact duration has only little effect on the brain.

3) Converting Severity Indices to the Abbreviated Injury Scale: Unfortunately, Severity Indices do usually not provide a direct scaling of injury but rather a limit between severe and non-severe injury. Furthermore, they are defined with respect to different physical domains and thus are not directly comparable to each other, nor can they be combined. In order to cope with this deficit, mappings were developed to translate a Severity Index to the Abbreviated Injury Scale. The NHTSA $^{8}$ specified the expanded Prasad/Mertz curves [17] for converting $\mathrm{HIC}_{15}$ values to the probability $p(A I S \geq i)$ of the corresponding AIS level $i$ which are shown in Fig.1(left). In [18] a conversion from $\mathrm{HIC}_{36}$ to $p(A I S \geq 2,3,4)_{\mathrm{HIC} 36}$ is defined. Since the EuroNCAP underlays its injury risk level definition mainly on the $p(A I S \geq 3)$-level, the corresponding functions for both HICs are illustrated in Fig.1(right):

$$
\begin{array}{r}
p(A I S \geq 3)_{\mathrm{HIC} 15}=\frac{1}{1+e^{3.39+\frac{200}{\mathrm{HIC}_{15}}-0.00372 \mathrm{HIC}_{15}}} \\
p(A I S \geq 3)_{\mathrm{HIC} 36}=\Phi\left(\frac{\ln \left(\mathrm{HIC}_{36}\right)-\mu}{\sigma}\right),
\end{array}
$$

with $\Phi($.$) denoting the cumulative normal distribution with$ mean $\mu=7.45231$ and standard deviation $\sigma=0.73998$. For our very short impacts the evaluation of $\mathrm{HIC}_{15}$ and $\mathrm{HIC}_{36}$ lead to the same numerical value. Obviously the $\mathrm{HIC}_{15}$ indicates a higher risk level than the $\mathrm{HIC}_{36}$ for the same numerical value and is therefore more restrictive.

\section{Injury Criteria For The Neck}

In general, the injury mechanisms of the human neck are related to forces and bending torques acting on the spinal column. In the EuroNCAP the corresponding limits are defined with respect to the positive cumulative exceedance time as denoted in Tab.III. Between these values a linear interpolation is carried out. The corresponding taxonomy of the neck is illustrated in Fig.2, whereas the EuroNCAP specifies limit values only for the motions listed in Tab.III.

\footnotetext{
${ }^{8}$ National Highway Traffic Safety Administration
}
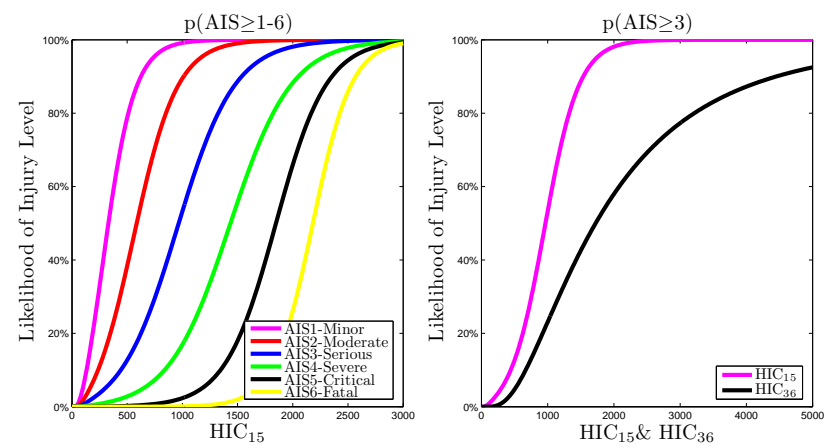

Fig. 1. Mapping $\mathrm{HIC}_{15}$ to the Abbreviated Injury Scale (left) and comparing $p(A I S \geq 3)_{\mathrm{HIC} 15}$ with $p(A I S \geq 3)_{\mathrm{HIC} 36}$ (right).

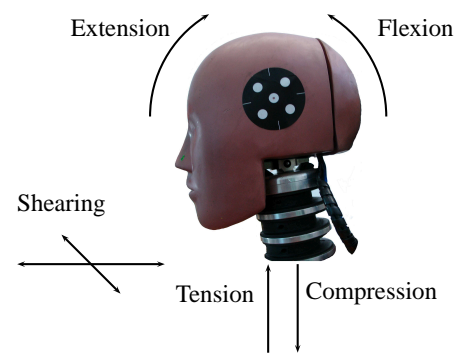

Fig. 2. Taxonomy of neck motions.

\section{E. Injury Criteria For The Chest}

1) Compression-Criterion: From evaluated cadaver experiments it was derived that acceleration and force criteria alone are intrinsically not able to predict the risk of internal injuries of the thorax which tend to be a greater threat to human survival than skeletal injury. Kroell analyzed a large data base of blunt thoracic impact experiments and realized that the Compression Criterion

$$
C C=\left\|\Delta \mathbf{x}_{C}\right\|_{2} \leq 22 \mathrm{~mm}
$$

is a superior indicator of chest injury severity, where $\Delta \mathbf{x}_{C}$ is the chest deflection. Especially sternal impact was shown to cause compression of the chest until rib fractures occur ([19], [20]).

2) Viscous-Criterion: The second criterion for the chest is the Viscous Criterion (VC), which is also known as Soft Tissue Criterion [20],[21]. It can be formulated as

$$
V C=c_{c}\left\|\Delta \dot{\mathbf{x}}_{C}\right\|_{2} \frac{\left\|\Delta \mathbf{x}_{C}\right\|_{2}}{l_{c}} \leq 0.5 \frac{\mathrm{m}}{\mathrm{s}},
$$

defined as the product of compression velocity and the normalized thoracic deflection. The scaling factor $c_{c}$ and the deformation constant (actually the initial torso thickness) $l_{c}$ depend on the used dummy and are summarized in [22].

\section{EXPERIMENTAL SETUP}

The instrumentation of the used HybridIII dummy is shown in Fig.3. It represents the standard equipment to measure the described injury criteria at a sampling frequency of $20 \mathrm{kHz}$. The signals are filtered according to [23]. 


\begin{tabular}{llll}
\hline Load & $@ 0 \mathrm{~ms}$ & $@ 25-35 \mathrm{~ms}$ & $@ 45 \mathrm{~ms}$ \\
\hline Shearing: $F_{x}, F_{y}$ & $1.9 / 3.1 \mathrm{kN}$ & $1.2 / 1.5 \mathrm{kN}$ & $1.1 / 1.1 \mathrm{kN}$ \\
Tension: $F_{z}$ & $2.7 / 3.3 \mathrm{kN}$ & $2.3 / 2.9 \mathrm{kN}$ & $1.1 / 1.1 \mathrm{kN}$ \\
Extension: $M_{y}$ & $42 / 57 \mathrm{Nm}$ & $42 / 57 \mathrm{Nm}$ & $42 / 57 \mathrm{Nm}$ \\
\hline
\end{tabular}

TABLE III

HIGHER AND LOWER PERFORMANCE LIMIT SPECIFIED FOR THE HUMAN NECK (SEE AS WELL FIG.8).

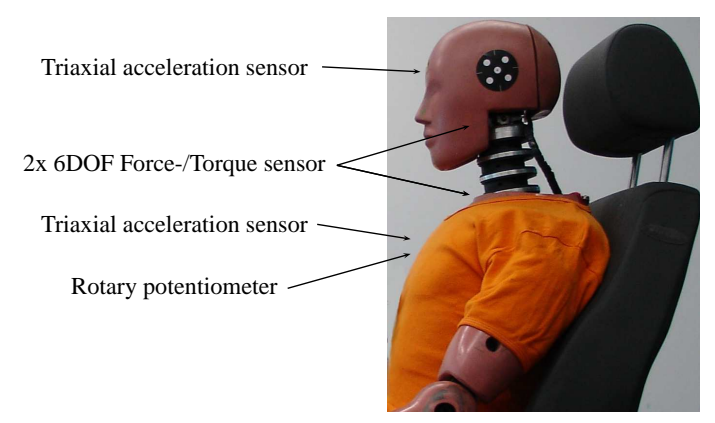

Fig. 3. HybridIII Dummy Instrumentation.

In Fig. 4 the overall test setup is shown. It consists of the LWRIII, the full dummy equipment, a high-speed camera and a laser pointer to ensure a reproducibility of the tests. The $7 \mathrm{DOF}^{9}$ flexible-joint robot has a weight of $14 \mathrm{~kg}$ and a load to weight ratio $\approx 1$. It is equipped with motor and link side position and torque sensors in each joint. All calculations of the indices were carried out by the ADAC, thus were done according to the EuroNCAP program. In order to ensure rigid and defined contact, a $1 \mathrm{~kg}$ aluminum impactor was used which was equipped with high bandwidth force and acceleration sensors (see Fig.4). The desired trajectory ${ }^{10}$ was a rest-to-rest motion which start and end configuration was given by

$$
\mathbf{q}_{\text {start }}=\left(\begin{array}{lllllll}
-45 & 90 & -90 & -45 & 0 & -90 & 147
\end{array}\right)^{\circ}
$$$$
\mathbf{q}_{\mathrm{end}}=\left(\begin{array}{rrrrrrr}
45 & 90 & -90 & 45 & 0 & -90 & 147
\end{array}\right)^{\circ} .
$$

In order to maximize the joint mass matrix (reflected inertia was $\approx 4 \mathrm{~kg}$ at the TCP) the trajectory was selected such that the robot hits the dummy in outstretched position. Furthermore, high TCP velocities can be achieved in this impact configuration. In our experiments we chose the robot velocities to be $\|\dot{\mathbf{x}}\|_{\mathrm{TCP}} \in\{0.2,0.7,1.0,1.5,2.0\} \mathrm{m} / \mathrm{s}$.

A TCP velocity of $2 \mathrm{~m} / \mathrm{s}$ is already relatively close to the maximal robot speed and, as will be pointed out later, poses in the case of impact a potential threat to the mechanics of the robot. Of course this position can be further optimized towards the absolute worst-case, but the described configuration seemed to be a reasonable first guess.

\section{EXPERIMENTAL RESULTS}

\section{A. Results for the Head}

In Fig. 5 the resulting $\mathrm{HIC}_{36}$ values are plotted with respect to the impact velocity of the robot. The corresponding injury classification was described in Sec.II-B. In order to classify an impact into the green labeled region, the occurring $\mathrm{HIC}_{36}$

\footnotetext{
${ }^{9}$ Degrees Of Freedom

${ }^{10}$ See also the video on www.robotic.dlr.de/safe-robot
}

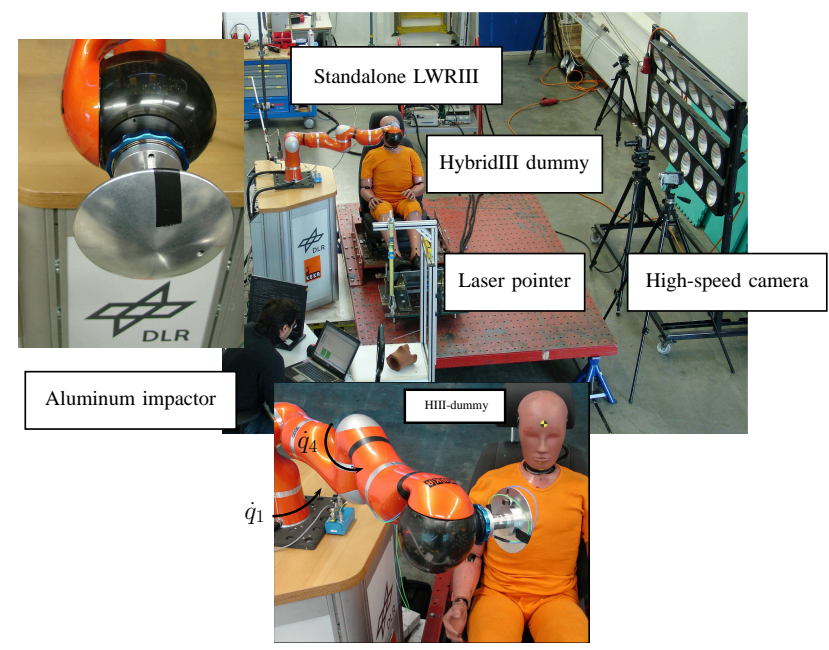

Fig. 4. Test setup for impact experiments.

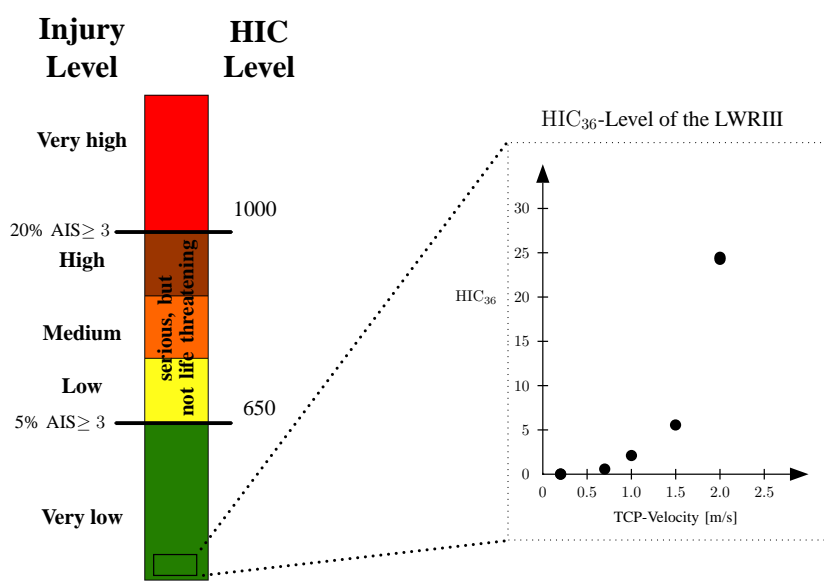

Fig. 5. Resulting $\mathrm{HIC}_{36}$ values for varying impact velocities, rated according to the EuroNCAP Assessment Protocol And Biomechanical Limits.

must not exceed 650 , which corresponds to a resulting 5\%probability of serious injury (AIS $\geq 3$ ). This value originates from [24], [25] and differs only slightly from the one obtained by the fitting function (4).

As indicated in Fig.5, the $\mathrm{HIC}_{36}$ caused by the LWRIII is below 25 at $2 \mathrm{~m} / \mathrm{s}$ which corresponds to a very low injury level. The resulting probability of injury severity obtained by (3) and (4) is $\approx 0 \%$ for all categories. Another aspect that clearly can be extracted from Fig.5 is that the $\mathrm{HIC}_{36}$ is rapidly increasing with robot velocity.

Similar to the results of the $\mathrm{HIC}_{36}$, very low potential danger is indicated by the $3 \mathrm{~ms}$-Criterion. Even at a tip velocity of $2 \mathrm{~m} / \mathrm{s}$ less than $20 \%$ of the lower limit of $72 \mathrm{~g}$ are reached (see Fig.6).

\section{B. Results for the Neck}

The resulting neck force $F_{\text {res }}^{\text {Neck }}$ for varying robot velocities caused by head impacts is illustrated in Fig.7. The actual impact is characterized by a very short peak which duration and maximum value depend on the impact velocity. For fast impacts a low level safety feature of the robot activates and 


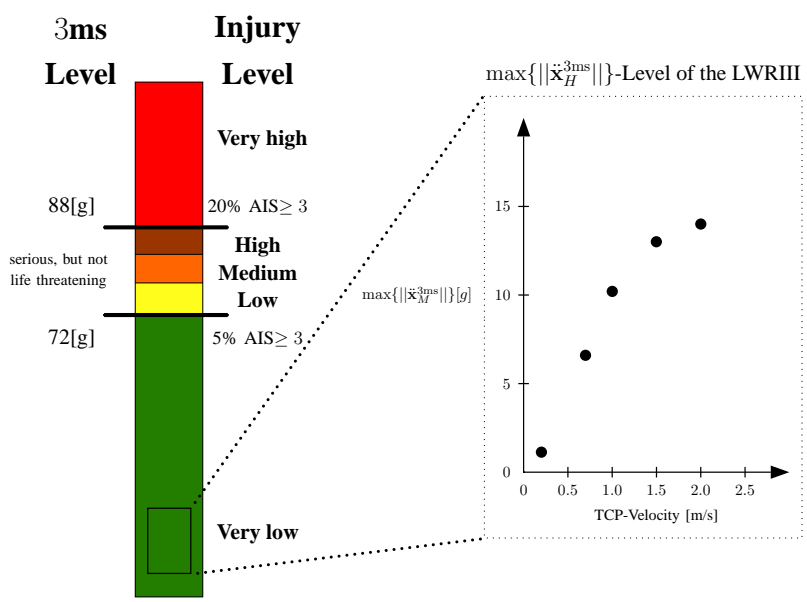

Fig. 6. Resulting 3ms-Criterion values for varying impact velocities, rated according to the EuroNCAP Assessment Protocol And Biomechanical Limits.

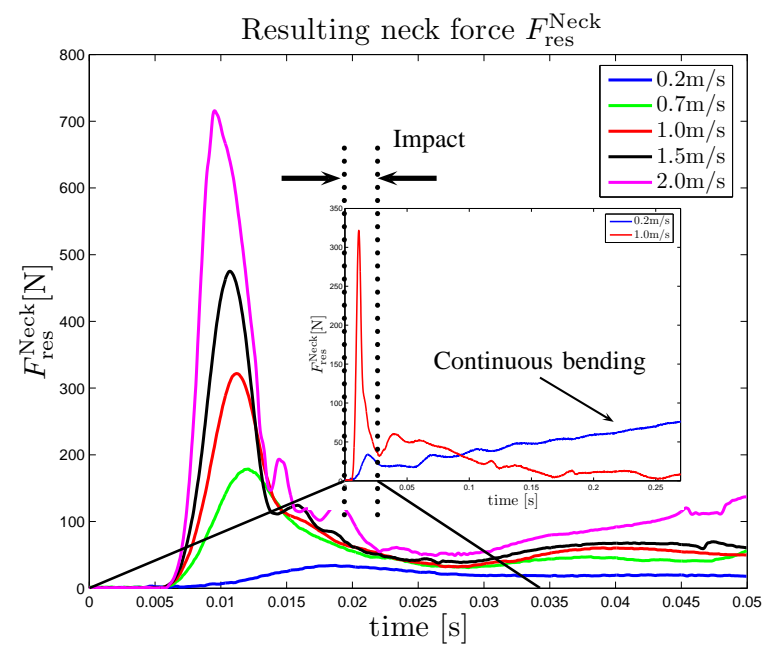

Fig. 7. Resulting impact force during head impacts.

stops it because the specified maximum joint torques are exceeded. Therefore, the maximum neck force/torque during the entire collision is determined by this peak force/torque occurring within the first $5-20 \mathrm{~ms}$ of the impact. On the other hand, if the impact velocity is very low $(0.2 \mathrm{~m} / \mathrm{s})$, the impact force is reduced dramatically and does not trigger the low-level stopping mechanism. Consequently, steadily growing neck bending can take place, increasing neck forces to even larger values than the ones caused by the original impact because the robot still follows its desired trajectory. This becomes clear if the neck forces for the impact velocities $0.2 \mathrm{~m} / \mathrm{s}$ and $1.0 \mathrm{~m} / \mathrm{s}$ are plotted for a longer time period (see Fig.7): After $\approx 20 \mathrm{~ms}$ both impact maxima are over and at $1 \mathrm{~m} / \mathrm{s}$ the low-level stop of the robot is triggered because the impact forces (up to $2 \mathrm{kN}$ were measured at the aluminum impactor) cause extremely high joint torques. In contrast at $0.2 \mathrm{~m} / \mathrm{s}$ the neck force is steadily increasing and might become even larger than impact forces at higher velocities.

In Fig.8 the occurring upper neck shearing and tension/compression forces are plotted with respect to the positive cumulative exceedance time. Actually, only tension limits are
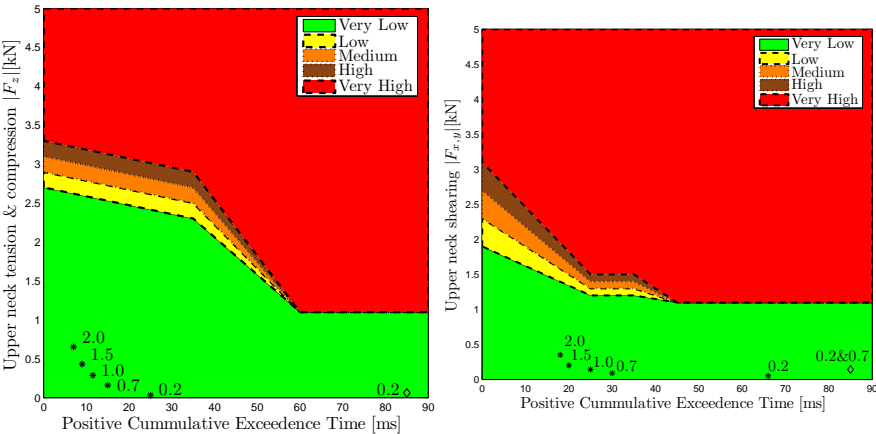

Fig. 8. Resulting $F_{x, y}$ and $F_{z}$ values for varying impact velocities, rated according to the EuroNCAP Assessment Protocol And Biomechanical Limits.

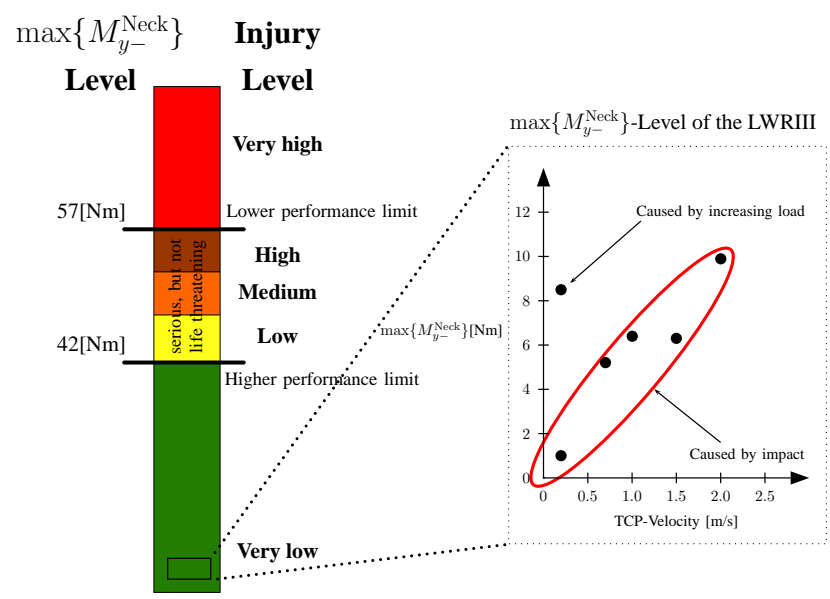

Fig. 9. Resulting $\max \left\{M_{y-}^{\mathrm{Neck}}\right\}$ values for varying impact velocities, rated according to the EuroNCAP Assessment Protocol And Biomechanical Limits.

specified in the EuroNCAP, but according to [26] tension is more critical than compression and thus applying available limits to both, tension and compression seems to be a reasonable choice.

The tolerance values for neck forces are not constant, but a function of the exceedance time (see Sec.II-D). The resulting forces are labeled with the corresponding TCP velocity. A $*$ indicates the forces caused by the impact and $\diamond$ the ones by continuous bending, if they were finally larger than the impact forces. In order not to break the dummy neck the robot stopped a predefined distance after the collision occurred. This of course limits the bending forces \& torques which otherwise would further increase. In Fig.9 the results of the extension torque are visualized. As for the previous head Severity Indices, the occurring neck forces/torques are totally subcritical, i.e. pose no threat to the human.

\section{Results for the Chest}

According to [27] a 5\%-probability of serious chest injury (AIS $\geq 3$ ) corresponds to a compression of $22 \mathrm{~mm}$ and $50 \%$ to $50 \mathrm{~mm}$. In Fig. 10 the resulting compression values are plotted with respect to the impact velocity of the robot. Again, the injury potential is very low, as the values range in the lowest quarter of the green area.

The results of the Viscous Criterion are not presented 


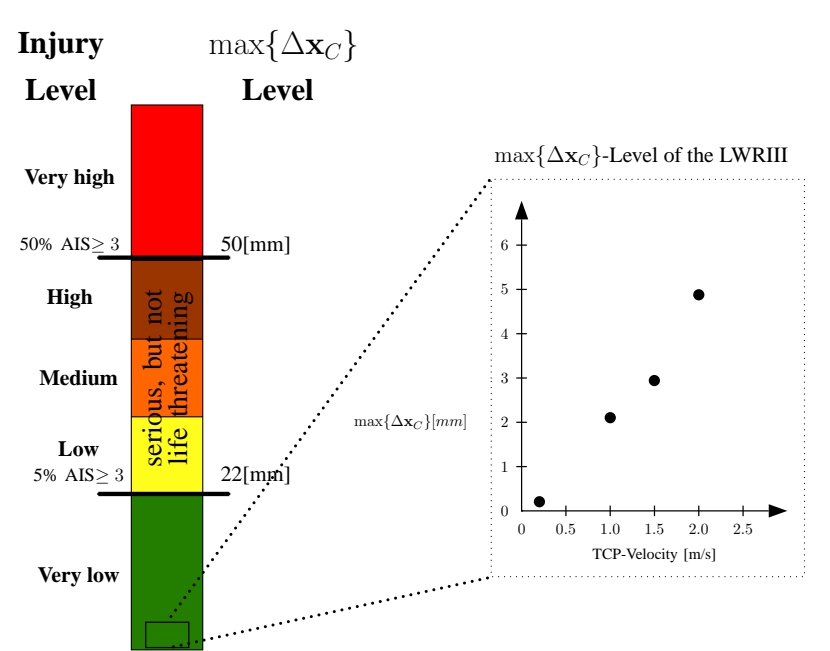

Fig. 10. Resulting $\max \left\{\Delta \mathbf{x}_{C}\right\}$ values for varying impact velocities, rated according to the EuroNCAP Assessment Protocol And Biomechanical Limits.

because the resulting values were located within the range of noise and thus this criterion is not well suited, nor sensitive enough for our evaluation. This is related to the relatively low velocities, compared to the ones encountered in automotive crashes.

\section{Evaluation \& Discussion}

All evaluated severity measures range within the lowest quarter of the green indicated area, i.e. the potential danger emanating from the LWRIII is intrinsically very low by means of injury measurable by automobile crash-test facilities such as the ADAC. These are surprising and gratifying results and to our knowledge, they represent the first systematic experimental evaluation of possible injuries during robot-human impacts using standardized testing facilities.

The low values of the Severity Indices are a direct consequence of the much lower speeds of the robot tip, compared to velocities of cars involved in crash-tests. There, impact tests at velocities starting at $10 \mathrm{~m} / \mathrm{s}$, which is equivalent to $36 \mathrm{~km} / \mathrm{h}$, are carried out. At such high velocities Severity Indices possibly exceed the defined limits.

Apart from the directly measured results one is able to draw fundamental implications to physical human-robot interaction, which now shall be outlined.

\section{A. Typical Impact Characteristics}

In order to illustrate certain problems and first implications resulting from the dynamics of a rigid head impact, a dummy head crash at $2 \mathrm{~m} / \mathrm{s}$ shall be evaluated. In Fig.11(left) the measured joint torque of the 4 th axis $\tau_{4}$, the contact force $\boldsymbol{F}_{\text {ext }}$, and impactor acceleration $\ddot{\boldsymbol{x}}_{\mathrm{Al}}$ are visualized. Such a fast impact is characterized by a very high acceleration/force peak, lasting $6-10 \mathrm{~ms}$ and highly depending on the impact velocity. The maximum measured contact force and acceleration of the aluminum impactor were $2 \mathrm{kN}$ and $35 \mathrm{~g}$, respectively. Actually, this very short part of the impact is the relevant one for the evaluation of head Severity Indices, since here they reach their maximum value.
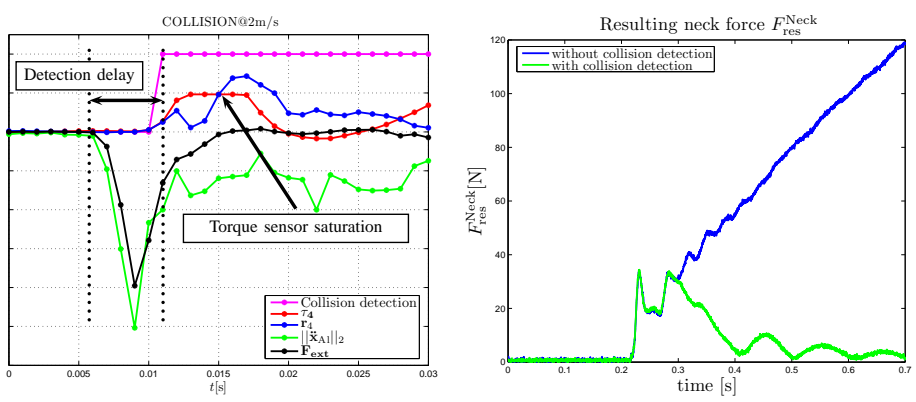

Fig. 11. Impact characteristics at $2 \mathrm{~m} / \mathrm{s}$. All values are scaled to fit into one plot (left). The plot is mainly to show the timing of the signals: While acceleration and impact force are simultaneous, the joint torque and the additional external torque estimation react delayed to the impact. Resulting neck force with and without collision detection and reaction strategy (right).

One clearly can see that before the joint torque starts increasing, the relevant force/acceleration peak period is practically over. Thus, during this particular time interval motor and link inertia are decoupled by the intrinsic joint elasticity, and only the link inertia is involved into the impact. Therefore, decreasing joint stiffness e.g. via antagonistic actuation would not have any effect on a hard contact head impact with link inertias similar or higher than the ones of the LWRIII. For collisions with softer body parts (e.g. the arm) the impact duration is lower and decreasing joint stiffness might reduce contact forces.

A collision detection and reaction scheme, based on the disturbance observer developed in [28], is used and indicated in Fig.11(left). It utilizes only the proprioceptive capabilities of the robot (joint torque and motor position) and provides a filtered version of the external torque $\tau_{\text {ext }}$. As soon as a collision has been detected (after a detection delay of $\approx 6 \mathrm{~ms}$ ) the robot switches within one cycle time of $1 \mathrm{~ms}$ from position to torque control with gravitation compensation [29]. In other words, the commanded torque is $\tau_{\mathrm{d}}=\bar{g}(\boldsymbol{\theta})$, where $\bar{g}$ is a gravitation compensation based on the motor position $\boldsymbol{\theta}$.

The disturbance observer highly depends on the joint torque measurement. This implies that the collision detection cannot be used to decrease the resulting injury severity caused by rigid impacts at high robot velocities, since the impact itself is passed before the joint torques start increasing significantly. Thus, using this collision detection mechanism does not clarify whether one could potentially decrease the resulting injury by a faster detection mechanism. Therefore, the acceleration signal of the impactor was used as well to trigger the reaction strategy of the robot. Although one could now detect the collision within $1 \mathrm{~ms}$, the resulting injury criteria did not differ from the ones obtained with the previous collision detection scheme or even without any reaction strategy. This is explained by the fact that, even if a collision is detected timely, the motors cannot revert their motion sufficiently fast.

Another fundamental injury source is quasistatic loading occurring at lower velocities (see Sec.IV-B), or if no lowlevel stop in case of maximum joint torque exceedance would occur and the robot continued to follow its desired trajectory after the impact. Especially if a human body part is clamped or lacks somehow in mobility, this could become a very dangerous injury source. In Fig.11(right) the effect of the 
collision detection is visualized which proves to be a very fast and efficient way to handle quasistatic loadings.

\section{B. Protecting The Robot}

Another very important observation, already made at an impact velocity of $1 \mathrm{~m} / \mathrm{s}$, is that the specified maximum joint torques of the robot were exceeded for several milliseconds during the impact (see Fig.11(left)).

A mechanical end stop in the robot limits the deflection range of the torque sensor which then goes into saturation. As already mentioned, a low-level emergency stop is initialized as soon as this event is triggered. It has been explained that even an ideally fast collision detection is not able to reduce the impact forces. Therefore, such dynamic loads pose a serious threat, potentially causing mechanical damage to the robot. This necessitates to think about how to prevent the robot from being damaged during such an impact, directly leading to the requirement of reducing the robot speed to subcritical values from the robot's point of view. One could even say that a rigid impact with the LWRIII poses a disproportionally higher threat to the robot than to the human.

\section{Influence of Robot Mass \& Velocity}
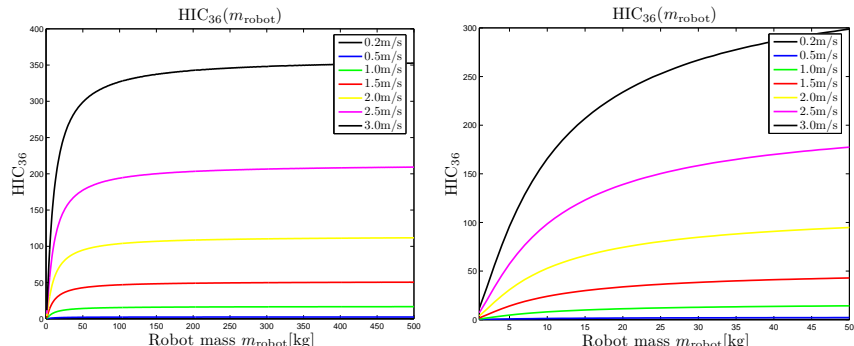

Fig. 12. HIC values resulting from 1DOF impact simulations between a robot and a dummy head model obtained by data fitting to real impact measurements. The curves show the dependency of HIC on the robot mass and are parameterized by the impact velocity.
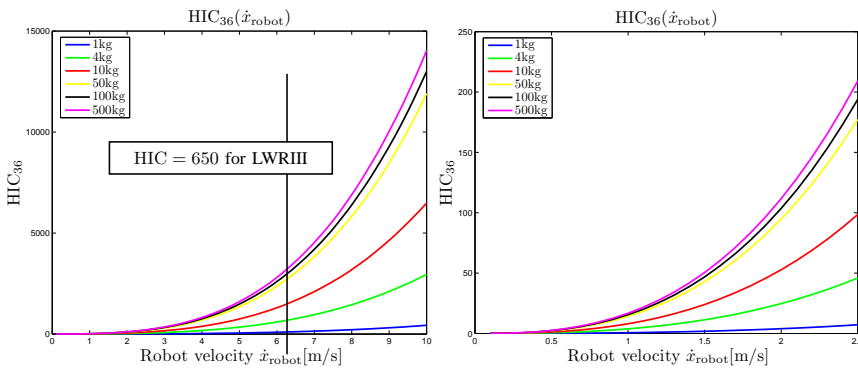

Fig. 13. HIC values resulting from 1DOF impact simulations between a robot and a dummy head model obtained by data fitting to real impact measurements. The curves show the dependency of HIC on the impact velocity and are parameterized by the robot mass.

In Fig.12,13 the simulation results of a robot colliding with a dummy head model, which was extracted from the real impact data, are plotted. A Hunt-Crossley model [30] was used to cope with the discontinuity in the classical mass spring damper approach caused by the damping element at the moment of impact. Although some variations between real experiments and this simulation may exist, very interesting and useful implications can be extracted from it. In Fig.12 the Head Injury Criterion was evaluated for robot masses up to $500 \mathrm{~kg}$ and graphs were obtained for impact velocities of $\|\dot{\mathbf{x}}\|_{\text {TCP }} \in\{0.2,0.5,1.0,1.5,2.0,2.5,3.0\} \mathrm{m} / \mathrm{s}$. They show that the HIC saturates for increasing robot mass at each impact velocity. This on the other hand indicates that at some point increasing robot mass does not result in higher HIC. In Fig.13 the vast effect of impact velocity is shown and additionally one can see the decreasing robot mass dependency. The impacts were simulated with robot velocities up to $10 \mathrm{~m} / \mathrm{s}$ and the graphs were obtained for reflected robot inertias of $\{1,4,10,50,100,500\} \mathrm{kg}$.

Additionally, a very intuitive and afterwards obvious interpretation of the saturation effect can be drawn: If we think of a very massive industrial robot, colliding at $2 \mathrm{~m} / \mathrm{s}$ with a human head it is nearly the same as if the human runs with $2 \mathrm{~m} / \mathrm{s}$, which is equivalent to $7.2 \mathrm{~km} / \mathrm{h}$, against a rigid wall. This is at least true for the short duration of the impact which is relevant for the HIC. Already intuitively it becomes clear that one would not be seriously injured by such an impact at walking speed.

Consequently, no robot whatever mass it has could become dangerous at $2 \mathrm{~m} / \mathrm{s}$ by means of the presented criteria as long as clamping and impacts with sharp surfaces can be excluded. In fact, even in case of clamping the Head Injury Criterion and the $3 \mathrm{~ms}$-Criterion would not indicate any potential injury. This is because they are determined within the first $5-10 \mathrm{~ms}$ of the impact and during this extremely short time interval the head does not move noticeable.

Generally speaking increasing robot mass and loads pose a threat if there is any chance a human body part could be clamped. Because of the higher inertias it would take the robot longer to decelerate and thus fatal injuries, e.g. exceeding chest deflection limits could be the consequence. This yields to another advantage of the LWRIII: By its lightweight design the inertias are low enough not to cause this kind of injury. In order to verify that statement, impact tests with industrial robots are going to be carried out, which probably yield similar HIC values as the ones obtained with the LWRIII, but the quasistatic contact force and penetration depth are going to increase significantly.

\section{Consequences for Standards \& Norms}

The presented results imply that typical Severity Indices, such as the Head Injury Criterion are not applicable to robot-human impacts occurring at much lower velocities than the ones evaluated in the automobile industry. Actually, it has been shown in [31] that the mechanical response of a dummy indicates even higher injury severity than would occur in reality. Therefore, new criteria focusing on other injury mechanisms, such as clamping, lacerations and fractures have to be investigated. These statements do not directly apply to robots operating at much higher velocities but it is questionable anyway whether it is desirable for a human to interact with a robot moving at velocities considerably higher than $2 \mathrm{~m} / \mathrm{s}$. Of course, another potential injury source still has to be investigated: Tools mounted on the robot can be arbitrarily dangerous and need to be investigated separately (what if the robot moves a knife). 
In the beginning of this paper the ISO-10218 was introduced, defining new collaborative operation requirements. In our experiments we were able to drive eight times faster and cause thirteen times higher dynamical contact forces than are suggested by the norm for the static case (Severity Indices are usually defined for impacts in the range of milliseconds but static tolerance is usually higher than dynamic [26]). Still our impact experiments yielded such low injury risks raising the question whether this standard is not too conservative.

\section{CONCLUSION \& OUTLOOK}

The motivation of this work was to investigate the potential danger emanating from the LWRIII with respect to physical human-robot interaction. Severity Indices were introduced, which are biomechanically motivated quantities indicating the injury severity of various human body parts. In this paper we focused on the blunt unexpected impact with the human standing still at the moment of impact. The important issue of tools still has to be investigated.

Impact tests were carried out using standard crash-test facilities, whereas numerous Severity Indices were evaluated according to the standardized EuroNCAP. The resulting values proved that a blunt impact between a human and the LWRIII does not cause serious harm ${ }^{11}$. Additionally it has been shown, that the results concerning the HIC can be generalized to robots of arbitrary mass.

Another major conclusion is that classical Severity Indices, established in the automobile industry cannot be transferred to the field of robotics because operating velocities are basically far too low to have any significant impact on them. Therefore, new criteria have to be proposed focusing on relevant injury mechanisms. Based on our results the adjustment or even redefinition of the automotive injury scaling system to robotic purposes will be of major importance.

A video illustrating and supporting the key aspects proposed and explained in the paper can be found at www.robotic.dlr.de/safe-robot

\section{ACKNOWLEDGMENT}

This work has been partially funded by the European Commission's Sixth Framework Programme as part of the projects SMERobot ${ }^{\mathrm{TM}}$ under grant no. 011838 and PHRIENDS under grant no. 045359 .

The authors would like to thank Andreas Ratzek and all involved members of the ADAC team for the fruitful and enjoyable cooperation. Furthermore, we would like to express our appreciation to Professor Dr. Dimitrios Kallieris for his helpful advise.

\section{REFERENCES}

[1] EN953, "Safety of Machinery - Guards - General Requirements for the Design and Construction of fixed and movable Guards," 1997.

[2] "http://www.phriends.eu/."

[3] "http://www.smerobot.org/."

\footnotetext{
${ }^{11} \mathrm{By}$ means of presented criteria. Furthermore, the presented evaluation is carried out for average male dummies and not for females or children. For such an analysis further tests with specially designed female or child dummies would be necessary.
}

[4] Ch. Ott, O. Eiberger, W. Friedl, B. Bäuml, U. Hillenbrand, Ch. Borst, A. Albu-Schäffer, B. Brunner, H. Hirschmüller, S. Kielhöfer, R. Konietschke, M. Suppa, T. Wimböck, F. Zacharias, and G. Hirzinger, "A Humanoid Two-Arm System for Dexterous Manipulation," in IEEE-RAS International Conference on Humanoid Robots, 2006.

[5] ISO10218, "Robots for industrial environments - Safety requirements Part 1: Robot," 2006.

[6] M. Zinn, O. Khatib, B. Roth, and J.K.Salisbury, "Playing It Safe Human-Friendly Robots," IEEE Robotics and Automation Magazine, vol. 11 , pp. 12-21, 2002.

[7] A. Bicchi and G. Tonietti, "Fast and Soft Arm Tactics: Dealing with the Safety-Performance Trade-Off in Robot Arms Design and Control," IEEE Robotics and Automation Magazine, vol. 11, pp. 22-33, 2004.

[8] H.-O. Lim and K. Tanie, "Human Safety Mechanisms of HumanFriendly Robots: Passive Viscoelastic Trunk and Passively Movable Base," International Journal of Robotic Research, vol. 19, no. 4, pp. 307-335, 2000.

[9] J. Heinzmann and A. Zelinsky, "Quantitative Safety Guarantees for Physical Human-Robot Interaction," International Journal of Robotic Research, vol. 22, no. 7-8, pp. 479-504, 2003.

[10] K. Ikuta, H. Ishii, and M. Nokata, "Safety Evaluation Method of Design and Control for Human-Care Robots," International Journal of Robotic Research, vol. 22, no. 5, pp. 281-298, 2003.

[11] J. Versace, "A Review of the Severity Index," Proc 15th Stapp Conference, vol. SAE Paper No.710881, 1971.

[12] M. Zinn, O. Khatib, and B. Roth, "A New Actuation Approach for Human Friendly Robot Design," The International Journal of Robotics Research, vol. 23, pp. 379-398, 2004.

[13] G. Tonietti, R. Schiavi, and A. Bicchi, Optimal Mechanical/Control Design for Safe and Fast Robotics, ser. Springer Tracts in Advanced Robotics, O. K. Marcelo H. Ang, Ed. Springer Berlin / Heidelberg, 2006, vol. 21.

[14] A. for the Advancement of Automotive medicine, The Abbreviated Injury Scale (1990) Revision Update 1998. Des Plaines/IL, 1998.

[15] EuroNCAP, "European Protocol New Assessment Programme - Assessment Protocol and Biomechanical Limits," 2003.

[16] J. McElhaney, R. Stalnaker, and V. Roberts, "Biomechanical Aspects of Head Injury," Human Impact Response - Measurement and Simulation, 1972.

[17] NHTSA, "Actions to Reduce the Adverse Effects of Air Bags," FMVSS No. 208, 1997.

[18] S. Kuppa, "Injury Criteria for Side Impact Dummies," NHTSA, 2004.

[19] I. Lau and D. Viano, "Role of Impact Velocity and Chest Compression in Thoracic Injury," Avia. Space Environ. Med., vol. 56, 1983.

[20] —, "The Viscous Criterion - Bases and Applications of an Injury Severity Index for Soft Tissues," Proceedings of 30th Stapp Car Crash Conference, vol. SAE Technical Paper No. 861882, 1986.

[21] _ " "Thoracic Impact: A Viscous Tolerance Criterion," Tenth Experimental Safety Vehicle Conference, 1985.

[22] D. P. V. S. Workgroup, "Crash Analysis Criteria Version 1.6.1," 2004.

[23] EuroNCAP, "European Protocol New Assessment Programme - Frontal Impact Testing Protocol," 2004.

[24] P. Prasad and H. Mertz, "The Position of the US Delegation to the ISO Working Group 6 on the Use of HIC in Automotive Environment," SAE Paper 851246, 1985.

[25] P. Prasad, H. Mertz, and G. Nusholtz, "Head Injury Risk Assessment for Forehead Impacts," SAE Paper 960099, 1985.

[26] D. Kallieris, Handbuch gerichtliche Medizin, B. Brinkmann and B. Madea, Eds. Springer Verlag, 2004.

[27] P. Prasad and H. Mertz, "Hybrid III sternal Deflection associated with Thoracic Injury Severities on Occupants restrained with Force-limiting Shoulder Belts," SAE Paper 910812, 1991.

[28] A. D. Luca, A. Albu-Schäffer, S. Haddadin, and G. Hirzinger, "Collision Detection and Safe Reaction with the DLR-III Lightweight Manipulator Arm," IEEE/RSJ International Conference on Intelligent Robots and Systems (IROS), pp. 1623-1630, 2006.

[29] C. Ott, A. Albu-Schäffer, A. Kugi, S. Stramigioli, and G. Hirzinger, "A Passivity Based Cartesian Impedance Controller for Flexible Joint Robots - Part I: Torque Feedback and Gravity Compensation," International Conference on Robotics and Automation (ICRA), pp. 2666-2672, 2004.

[30] K. Hunt and F. Crossley, "Coefficient of Restitution Interpreted as Damping in Vibroimpact," ASME Journal of Applied Mechanics, pp. 440-445, 1975.

[31] A. Rizzetti, D. Kallieris, P. Schiemann, and P. Mattern, "Response and Injury of the Head-Neck Unit during a Low Velocity Head Impact," IRCOBI, pp. 194-207, 1997. 